DOCTRINA

\title{
Abogacía laboral 4.o: Inteligencia artificial y problemáticas profesionales en la abogacía brasileña
}

\author{
Labor advocacy 4.0: Artificial intelligence and professional problems in Brazilian law \\ pratice
}

\author{
Rodrigo Monteiro Pessoa \\ Universidad de la Frontera, Chile
}

RESUMEN Recientemente, la noticia en Brasil del primer software de inteligencia artificial destinado a contestar dudas laborales y captar clientes encendió la discusión sobre la legalidad y la ética en el uso de estas herramientas tecnológicas. Si bien es cierto que cada vez más la tecnología invadirá los espacios de todas las profesiones, es necesario verificar la forma en que ésta se utiliza en el ejercicio de la profesión jurídica, principalmente en el caso del derecho laboral, que lidia con valores pertenecientes - la mayoría de las veces- al mínimo necesario para la sobrevivencia de las personas y sus familias. Este artículo estudiará el caso de consultoría laboral por la inteligencia artificial y sus posibles consecuencias éticas y profesionales en Brasil.

PALABRAS CLAVE Derecho laboral, inteligencia artificial, ética profesional.

ABSTRACT Recently, the news in Brazil of the first artificial intelligence software aimed at answering labor questions and attracting clients ignited the discussion about legality and ethics in the use of these technological tools. While it is true that more and more technologies will invade the spaces of all professions, it is necessary to verify the way they're being used in the exercise of the legal profession, mainly in the case of Labor Law, which deals with values belonging to -most often- the minimum necessary for the survival of people and their families. This article will study the case of labor consultancy for artificial intelligence and its possible ethical and professional consequences in Brazil.

KEYWORDS Labor law, artificial intelligence, professional ethics. 


\section{El caso de «Valentina, el robot del trabajador»}

El 18 de mayo de 2018, la empresa Hurst Capital Ltda. lanzó en la red social Facebook un software de inteligencia artificial destinado a contestar dudas laborales de cualquier usuario en Brasil que quiera hacerlas a través de un chat en línea. La herramienta fue bautizada como «Valentina, el robot del trabajador» (Valentina, o robô do trabalhador) y fue responsable por encender, aún más, la polémica sobre el uso de la inteligencia artificial en la abogacía. ${ }^{1}$ La misma empresa ya estaba utilizando otras dos páginas de chatbot en la abogacía: «Haroldo, el robot del consumidor», desde el 27 de febrero de 2018, y «Leopoldo, el robot del contribuyente», desde el 1 de marzo de 2018, lo que ya había generado un sinfín de análisis e insatisfacción por parte de los abogados brasileños. ${ }^{2}$

En la nota de la OAB-RJ (Ordem dos Advogados do Brasil, Seccional do Rio de Janeiro), en conjunto con el IAB (Instituto dos Advogados Brasileiros), se repudia que el perfil del robot en Facebook «Valentina», además de asumir que no es abogada, afirma que puede «comprar la pelea», asumiendo los costos procesales y devolviendo los valores debidos en razón de deudas laborales por parte del empleador, quedándose con una «pequeña cuota» por esta actuación. El robot de Hurst ofrece una solución completa, al adquirir los derechos patrimoniales del empleado para actuar en su nombre en las vías administrativa y judicial.

La iniciativa de Hurst Capital derivaría del final de la gratuidad de las acciones laborales que, a su vez, redujeron en $56 \%$ (promedio nacional) las demandas judiciales en diciembre de $2017,{ }^{3}$ cuando entró en vigencia la Reforma Laboral en Brasil (Arese, Gunther y Toledo Filho, 2017; Martinez, 2018; Souza Júnior y otros, 2018). El robot ayudaría a los trabajadores a buscar la reparación e indemnización de derechos no cumplidos por los empleadores, admitiendo que la reducción de las acciones judiciales tendría como justificación el temor de buscar la justicia del trabajo, sea por la nueva onerosidad del proceso, sea por la falta de información que tienen los trabajadores.

Para ambas instituciones, el Estatuto de la Abogacía (Ley 8.906 de 1994) es taxativo al definir en el artículo primero que la postulación a un órgano del Poder Judicial y

1. Véase «Valentina - Robô do trabalhador», página de Facebook, disponible en http://bit.ly/2wIxHg8.

2. Véase, por ejemplo, «Entidades de advogados reagem a robô que ajuda em ações trabalhistas», Consultor Jurídico, 1 de julio de 2018, disponible en http://bit.ly/2wExhYj; Fabio Gomes, «Advogados reagem a robô que ajuda em ações trabalhistas», Jusbrasil, julio de 2018, disponible en http://bit.ly/2wGAuqs; Anelly Medeiros, «Entidades repudiam substituição de advogados por robôs na internet», Poder Judiciário, 2 de julio de 2018, disponible en http://bit.ly/2wG2oTq, además de la nota conjunta de la Orden de los Abogados Brasileños de Río de Janeiro y del Instituto de los Abogados Brasileños, disponible en http://bit.ly/2EXV4Hf.

3. Fernando Martines, «Ações trabalhistas caem em todos os TRTs, mas advogados apontam represamento», Consultor Jurídico, 7 de febrero de 2018, disponible en http://bit.ly/2wGAhmY. 
a los juzgados especiales, así como las actividades de consultoría, asesoría y dirección jurídicas son actividades privativas de los abogados. ${ }^{4}$ Por esta razón, están en contra del sistema alternativo de solución privada de acceso a la justicia, que se aprovecha de los trabajadores, explotando uno de los efectos más dañinos causados por la llamada reforma laboral, que es el acceso a la justicia laboral especializada por aquellos que dependen de su gratuidad.

En respuesta a la nota de prensa de la OAB-RJ y del IAB, la empresa Hurst Capital emitió un comunicado que aclara su posición, en el que afirma que utiliza la inteligencia artificial -incluso robots de chat- para localizar y adquirir derechos patrimoniales disponibles a través de la cesión de crédito, que es una modalidad de transmisión de la obligación prevista en el artículo 286 del Código Civil brasileño. ${ }^{5}$ Luego de adquirir los derechos del trabajador, hace negociaciones administrativas y contrata a los abogados para iniciar los procedimientos judiciales contra los deudores. Por lo tanto, para todos los procedimientos jurídicos o que involucren al Poder Judicial, el trabajo vinculado con la función de abogado es efectuado por oficinas especializadas en cada materia. En este sentido, la empresa manifiesta su entera concordancia y cumplimiento con lo que determina la Constitución Federal brasileña de 1988, ${ }^{6}$ las leyes infraconstitucionales y, especialmente, el Estatuto de la Abogacía:

La empresa Hurst Capital Ltda. entiende que las innovaciones tecnológicas pueden ser mal comprendidas y causar cierta polémica al principio, pero cree fuertemente que su tecnología facilita el acceso a la justicia, con la actuación saludable de los abogados, que son indispensables, y en beneficio del desahogo del Poder Judicial. ${ }^{7}$

La inteligencia artificial, en la conversación por chat con el trabajador, identificará la viabilidad de la demanda y el contenido económico de la misma, como un filtro de las opciones más rentables para la empresa, de forma que - y esto es una opinión nuestra - valga la pena movilizar el equipo entero para buscar una solución para el caso concreto. Esto es de fácil comprensión, ya que el propio sitio web de la empresa explica el uso de sus tecnologías, con los chatbots (robots de conversación)

4. «Lei 8.906, de 4 de julho de 1994, que dispõe sobre o Estatuto da Advocacia e a Ordem dos Advogados do Brasil (OAB)», Planalto, disponible en http://bit.ly/2wLrgJs.

5. Artículo 286 del Código Civil brasileño (Ley 10.406 de 2002): «El acreedor puede ceder su crédito si a esto no se opusiere la naturaleza de la obligación, la ley, o la convención con el deudor; la cláusula prohibitiva de la cesión no podrá oponerse al cesionario de buena fe si no figura en el instrumento de la obligación» (traducción nuestra del original en portugués).

6. Artículo 133 de la Constitución brasileña de 1988: «El abogado es indispensable para la administración de la justicia, siendo inviolable por sus actos y manifestaciones en el ejercicio de la profesión, dentro de los límites de la ley».

7. Hurst Capital Ltda., «Reposta à nota conjunta do IAB e da OAB/RJ», Consultor Jurídico, 29 de junio de 2018, disponible en http://bit.ly/2X1j3Qh. 
como aquéllos que «se comunican, por medio de procesamiento de lenguaje natural, con potenciales clientes en redes sociales en la búsqueda de demandas que aún no ingresan en el Poder Judicial», y los crawlers (robots de investigación) como aquéllos que «buscan en diarios oficiales y sitios de tribunales, utilizando técnicas de machine learning, e identifican procesos que posean potencial de retornos adecuados a nuestra política de inversión». ${ }^{8}$

Los problemas generados por esta tecnología se concentran básicamente en tres principales: i) la consultoría laboral por una inteligencia artificial, que según el Estatuto de la Abogacía de Brasil alude a la exclusividad de esta actividad por un profesional habilitado al ejercicio de la profesión; ii) la captación de acciones por medio de las redes sociales, que es igualmente prohibida por el Código de Ética y Disciplina de la OAB (Moraes, 2003: 519); ${ }^{9}$ y c) el posible menoscabo al trabajador en la obtención de los créditos laborales que les son debidos por la transferencia de parte o la totalidad de la indemnización a la empresa contratada, o por el uso de la solución extrajudicial del conflicto. Estos son los puntos que analizaremos en seguida.

\section{La consultoría laboral por un chatbot y las competencias privativas del abogado}

Para poder contextualizar la discusión, es trascedente aclarar al lector que el ejercicio de la abogacía en Brasil depende la inscripción en la OAB (Ordem dos Advogados do Brasil), que -según el artículo 44 de la Ley 8.906 de 1994- es un servicio público, dotado de personalidad jurídica propia y de forma federativa, y que tiene por finalidad defender la Constitución, el orden jurídico del Estado democrático de derecho, los derechos humanos y la justicia social, y pugnar por la buena aplicación de las leyes, la rápida administración de la justicia y el perfeccionamiento de la cultura y de las instituciones jurídicas; y promover, con exclusividad, la representación, la defensa, la selección y la disciplina de los abogados en toda la República Federativa del Brasil. La OAB no mantiene con órganos de la Administración Pública ningún vínculo funcional o jerárquico. Por lo tanto, la entidad es una persona jurídica sui generis, por prestar servicio público de forma institucional sin insertarse en la Administración Pública, directa o indirectamente (Piovezan y Oliveira Freitas, 2015: 357).

Todo y cualquier licenciado en ciencias jurídicas debe someterse al «examen de

8. Véase el sitio de la empresa en https://hurst.capital/.

9. En Brasil, la función de abogado es considerada una función de utilidad pública, no se trata de una actividad mercantil. La Constitución Federal de 1988 determina en el artículo 133 que el abogado es indispensable para la administración de la justicia. Por esta razón, el Código de Ética y Disciplina de la OAB prohíbe la publicidad, que es típica de empresas privadas y la captación de clientes, en su artículo 7: «Se prohíbe el ofrecimiento de servicios profesionales que impliquen, directa o indirectamente, inculcación o captación de clientela». 
orden», que es promovido por la propia $\mathrm{OAB}$, para poder habilitarse como abogado en el país. Este examen es nacional y permite a los licenciados en derecho el libre ejercicio en todo el territorio nacional. La importancia de aclarar esto es que el abogado en tierras brasileñas está obligatoriamente vinculado a la $\mathrm{OAB}$, que regula y fiscaliza el ejercicio de la profesión. Su estatuto y su Código de Ética y Disciplina deben ser cumplidos rigurosamente so pena de sanciones. El artículo 35 del Estatuto de la Abogacía determina que las sanciones van desde la censura hasta la exclusión, lo que inhabilitaría el ejercicio de la profesión de abogado.

$\mathrm{La} \mathrm{OAB}$ no es un colegio de abogados, con características gremiales y cuya afiliación es voluntaria como se da en el caso chileno, por ejemplo. ${ }^{10}$ Todos los que eligieron el libre ejercicio deben superar el examen de orden para estar vinculados a ella y deben cumplimiento a las leyes que fueron promulgadas para regular y fiscalizar este ejercicio. No es la Corte Suprema (Supremo Tribunal Federal en el caso brasileño) la que otorga el título de abogado en Brasil, es la $\mathrm{OAB}$ la que habilita a los profesionales titulados por sus universidades para este ejercicio.

Esta introducción es fundamental para este primer punto a analizar, ya que la Ley 8.906 de 1994 (Estatuto de la Abogacía en Brasil) es clara al determinar que son actividades privativas de la abogacía las de consultoría, asesoría y dirección jurídicas (artículo 1, inciso segundo). Aquí tenemos un gran problema con el chatbot «Valentina, el robot del trabajador», pues, a través de su banco de datos de información y su inteligencia artificial que permitirá a «Valentina» aprender constantemente sobre la forma de conectar su base de datos a las necesidades del «cliente», reflejan una clara modalidad de consultoría y asesoría jurídicas. En este caso, podríamos decir que la empresa que lo utiliza no está habilitada para el ejercicio de la profesión de abogado.

El trabajador que tiene algún problema en su relación de trabajo o por el término de su relación de trabajo, contará su caso a través de mensajes en la red social como si estuviese hablando con cualquiera de sus contactos en esta plataforma, $\mathrm{y}$ «Valentina» le instruirá y analizará el caso, que es elegido por la empresa Hurst como un caso a ser tomado, según la razonabilidad de la demanda. Si dentro de los parámetros de interés de la empresa el caso narrado por el trabajador es económicamente viable y rentable, «Valentina» ofrecerá los servicios de Hurst. El trabajador deberá aceptar los términos de uso del servicio, que transfieren parte del derecho de la indemnización o, si lo desea, su totalidad a la empresa contratada para litigar en su nombre. Todo esto se contrata electrónicamente. A partir de ahí, el equipo de Hurst asume todo el proceso, desde la contratación de abogados y su presencia en las audiencias pertinentes, hasta los costos de litigación. En caso de que exista alguna condenación en costas judiciales, la empresa afirma que asume estos gastos.

10. Véase el estatuto del Colegio de Abogados de Chile, aprobado por el Oficio 2.503 del Ministerio de Economía, Fomento y Reconstrucción, del 26 de abril de 1991. 
A primera vista, Hurst Capital estaría incurriendo en una contravención penal, que el artículo 47 del Decreto Ley 3.688 del 3 de octubre de 1941 en Brasil establece como «ejercer profesión o actividad económica o anunciar que la ejerce sin cumplir las condiciones a que por ley está subordinado su ejercicio».

No obstante, tenemos que pensar que, si la base de datos de consultoría de «Valentina» fue alimentada por abogados habilitados para el ejercicio de la profesión, y que un grupo de abogados igualmente da seguimiento a la forma en que esta inteligencia artificial va conectando su información con las preguntas de los clientes, entonces la empresa no estaría incurriendo en la contravención penal anteriormente citada.

Afirmamos esto porque «Valentina» no es un sujeto, es una herramienta. La inteligencia artificial puede ser definida hoy como una simulación de la inteligencia humana en una máquina, con el objetivo de hacerla eficiente para identificar y usar los fragmentos correctos del conocimiento para solucionar un problema (Konar, 1999: 3). Los sistemas recientes de inteligencia artificial necesitan, para la generación de explicaciones, de una estructura lógica subyacente al proceso de generación, es decir, para explicar algo necesita una base de conocimiento para hacer inferencias extraídas de esa base de conocimiento (Walton, 2005: 177). Si esta base de conocimiento es dada por abogados, se podría argumentar que los profesionales están utilizando una herramienta para facilitar el trabajo que ellos mismos están habilitados a hacer en persona. Pero lo hacen a través de una plataforma tecnológica, en una red social, por medio de un chatbot.

Este entendimiento es similar al proferido en el voto de la OAB/SP cuando fue consultada sobre el software de inteligencia artificial ELI (enhanced legal intelligence). ${ }^{11}$ La consulta a la OAB/SP número E-4.88o/2017, con voto proferido por los abogados Sérgio Kehdi Fagundes, Fábio Teixeira Ozi y Pedro Paulo Wen del Gasparini, comentó que el «robot abogado» ELI

se presta, según lo divulgado, a auxiliar a los abogados a ser más eficientes en sus actividades profesionales, sin suprimir el poder decisorio y las responsabilidades del profesional y, en este exclusivo sentido, aunque más sofisticado, la plataforma se une a tantas otras soluciones o herramientas utilizadas para el mismo fin, cuya falta en los días de hoy sería impensable. Dentro de esa perspectiva, las innovaciones tecnológicas con miras a auxiliar al abogado en el ejercicio de sus funciones no encuentran óbices legales y éticos.

Si esto es así, la empresa por detrás de la inteligencia artificial no vulneraría una competencia exclusiva de los profesionales jurídicos brasileños. Al contrario, la exis-

11. ELI es un robot asistente de abogados definido por la empresa como un asistente jurídico de alta performance que ayuda a los abogados, oficinas de abogados y empresas en problemas específicos con alta ganancia en productividad y calidad, lo que permite lograr excelentes resultados. 
tencia un nicho de mercado exigido hoy día por los profesionales jurídicos, cual sea la lógica jurídica, es cada vez más demandada para la implementación de argumentaciones válidas en materia de inteligencia artificial (Walton, 2005: xi). Se hace necesaria la presencia de los abogados para poder alimentar los algoritmos que permiten a la inteligencia artificial proceder con su proceso de inferencia de forma eficiente, conectada a la lógica legal del lugar en que actúa la inteligencia artificial. Por ende, nos lleva a pensar que esta situación configuraría mucho más una demanda por profesionales para generar una herramienta efectivamente útil, que el ejercicio ilegal.

No obstante, esto no termina ahí. Aunque supusiéramos que la empresa dispone de un equipo fijo de abogados que podría ser responsable por la alimentación de la base de conocimiento de «Valentina», y que estarían utilizando esta herramienta para facilitar su propia labor - lo que aparentemente no lo es, ya que la empresa, tomando casos a nivel nacional, tendrá que subcontratar abogados para atender a todas estas demandas-, la profesión de abogado en Brasil no puede ser mercantilizada. La permisibilidad del uso de la inteligencia artificial como conexión entre la empresa y los «clientes» vulneraría la intención de la norma que regula la profesión en el país. Explica Paulo Lôbo que

la consultoría jurídica no puede considerarse como oferta al público, de modo impersonal, por utilización de medios de comunicación como el teléfono o internet. El modelo de sociedad de abogados adoptado por la Ley 8.906/94 es el de organización de medios, no pudiendo tener fines mercantiles o empresariales (Lôbo, 2007: 28).

Esta posición fue corroborada por el Consejo Federal de la OAB, en la consulta 147/97/OEP, publicada en el Diario de Justicia de la Unión el 24 junio de 1997. Y es igualmente corroborada por otros autores (Piovezan y Oliveira Freitas, 2015: 14). Ergo, aunque exista un equipo responsable por la generación de la base de conocimiento que posibilita las inferencias de "Valentina», y la demanda por profesionales que puedan comprender la lógica jurídica como forma de alimentar las bases de una inteligencia artificial con miras a un trabajo eficiente frente a un dado ordenamiento jurídico, la regulación actual imposibilita el uso de esta herramienta para la consultoría jurídica, pues es una consultoría impersonal, prohibida por el Estatuto de la Abogacía.

Por ende, se trata de un caso diferente al caso juzgado por la OAB/SP (E$4.880 / 2017$ ) con la herramienta ELI, ya que ésta pretende servir de soporte para los propios abogados, como forma de hacer su trabajo más fácil y eficiente (recolección de datos, organización de documentos, ejecución de cálculos, formateo de escritos jurídicos, seguimiento de clientes y la rutina de procesos, asesoramiento en colaboraciones, informes inteligentes e interpretación de decisiones judiciales, entre otras actividades que aumentan la productividad del abogado). La herramienta ELI no tiene por objeto prestar una consultoría pública impersonal, ni tampoco captar clientes. 
Su uso se limita a optimizar el trabajo del abogado y no se destina a interactuar con el público como forma de contestar dudas sobre la relación de trabajo - como es el caso de «Valentina»—, o conocer de problemas jurídicos y contactar el cliente que buscó una consultoría a través del chatbot.

La función principal de «Valentina» es establecer una capacidad de predicción ofertando un servicio impersonal al público. La inteligencia artificial hará la predicción a través de algunos métodos que permiten encontrar la solución de un problema y predecir la probabilidad de que la respuesta sea favorable, además de medir la rentabilidad que la empresa puede obtener actuando en este caso.

Para esto, los algoritmos de la inteligencia artificial comprenden el $k$-means y el $c$ means, también conocido como agrupamiento difuso o fuzzy. El k-means fragmenta el problema en partes más simples, para poder solucionarlo de manera eficiente. El algoritmo fragmenta un objeto de observación en grupos y basado en la descripción encaja el objeto observado en uno de los grupos que componen su algoritmo (Benítez, y otros, 2014: capítulo 2, sección 3.4). El fuzzy o c-means complementa el algoritmo $k$-means de forma que se considere que el objeto de análisis igualmente puede pertenecer a otros grupos del cluster por sus elementos (Benítez, y otros, 2014: capítulo 2, sección 3.5). Por ende, el problema del agrupamiento difuso se reduce a encontrar una caracterización de este tipo que sea óptima.

Con este método, los datos que son informados por los trabajadores en el chat de Facebook permitirá que la inteligencia artificial analice el caso y determine la posibilidad de que la pretensión tenga un resultado favorable. La empresa que maneja la inteligencia artificial tiene finalidad lucrativa y necesita saber que el trabajador que está interactuando con su chatbot tiene un alto porcentaje de salir vencedor en una demanda. De ahí el porqué de considerar que la empresa visualizará esta situación como una probable «mercancía» que puede ser negociada por el interesado.

Dado el análisis anterior, aunque podemos decir que no existe una contravención penal por el uso de la inteligencia artificial por la empresa, la oferta impersonal de consultoría y la mercantilización de la profesión no son permitidas por el ordenamiento jurídico brasileño. Por lo que, frente al primer problema analizado, somos partidarios de la vulneración de las garantías de la profesión de abogado en Brasil en este primer momento.

\section{La captación de clientes y acciones por medio de las redes sociales y su prohibición por el Código de Ética y Disciplina de la OAB}

De los tres problemas planteados para nuestro análisis en esta investigación, el primero de ellos ya tiene una opinión. La consultoría prestada por las redes sociales vía chatbot vulnera las garantías de la abogacía, que no puede ser mercantilizada, al ser considerada como una oferta al público de un servicio jurídico impersonal por 
medio de internet. Comprender esta lógica es trascendente para estudiar el siguiente problema: la captación de clientes por los profesionales en el ejercicio de la profesión de abogado.

El Código de Ética y Disciplina de la OAB, Resolución 02/2015, promulgada dentro de las facultades de los artículos $33^{12}$ y 54 , inciso quinto ${ }^{13}$ del Estatuto de la OAB (Ley 8.906/94) prohíbe la publicidad, que es típica de empresas privadas y la captación de clientes, en su artículo 7: «Se prohíbe el ofrecimiento de servicios profesionales que impliquen, directa o indirectamente, inculcación o captación de clientela».

Igualmente, el Estatuto de la OAB (Ley 8.906/94), en su capítulo 9, "De las infracciones y sanciones disciplinares», artículo 34, alude que constituye infracción disciplinar (inciso cuarto) captar causas, con o sin la intervención de terceros. La captación es el acto de atraer o capturar a alguien con alguna finalidad. En el caso de nuestro estudio, es el acto de atraer o capturar clientes ofreciéndoles servicios de abogacía.

Dentro de la lógica de la profesión de abogado en Brasil, no se puede mercantilizar la profesión de forma que aquéllos que tienen más recursos puedan utilizar las herramientas de marketing a su favor para atraer más clientes que los demás profesionales. Por esta razón, el cliente debe buscar al abogado y no lo contrario (Lôbo, 2007: 204). Eso no significa que todo tipo de publicidad esté prohibida para los abogados, sino que ellos deben adecuar la forma en que llevarán su nombre al conocimiento del público, siempre siguiendo los parámetros ético-estatutarios (Baroni, 2009: 75).

El Consejo Federal de la OAB, a través del Proveimiento 94/2000, aclaró qué tipo de publicidad puede ser utilizada como medios lícitos en su artículo 3:

i) la utilización de tarjetas de visita y de presentación de la oficina, conteniendo, exclusivamente, informaciones objetivas; ii) la placa identificativa de la oficina, fijada en el lugar donde se encuentra instalada; iii) el anuncio de la oficina en listas telefónicas y análogas; iv) la comunicación de cambio de dirección y de alteración de otros datos de identificación de la oficina en los diversos medios de comunicación escrita, así como por medio de correo directo a los colegas y a los clientes registrados; v) la mención de la condición de abogado y, en su caso, del área de actuación, en anuarios profesionales, nacionales o extranjeros; vi) la divulgación de las informaciones objetivas, relativas al abogado o a la sociedad de abogados, con modicidad, en los medios de comunicación escrita y electrónica. Sección 1: La publicidad debe ser

12. Artículo 33: «El abogado se obliga a cumplir rigurosamente los deberes consignados en el Código de Ética y Disciplina. Párrafo único: El Código de Ética y Disciplina regula los deberes del abogado frente a la comunidad, el cliente, y otro profesional, y aún la publicidad, la rehúsa de representación, el deber de asistencia jurídica, el deber general de urbanidad y los respectivos procedimientos disciplinares».

13. Artículo 54: «Es de competencia del Consejo Federal: [...] 5) editar y alterar el Reglamento General, el Código de Ética y Disciplina, y los proveimientos que juzgue necesarios». 
hecha con discreción y moderación, observado lo dispuesto en los artículos 28, 30 y 31 del Código de Ética y Disciplina. Sección 2: Las cartas con base de datos y las tarjetas de presentación solo pueden ser suministradas a colegas, clientes o a personas que los soliciten o los autoricen previamente. Sección 3: Los anuncios de publicidad de servicios de abogacía siempre deberán indicar el nombre o el nombre social del abogado o de la sociedad de abogados con el respectivo número de inscripción o de registro; debe estar escrito en portugués o, si en otro idioma, ir acompañada de su respectiva traducción (véase el Proveimiento 172/2016).

De esta forma, se aclara que cualquier uso de publicidad que tenga por objetivo captar clientes de forma mercantilizada está prohibido. Aquí visualizamos perfectamente el uso a que se destina las herramientas desarrolladas por Hurst Capital, ya que su chatbot de consultoría en derecho laboral se publicita a través de una red social muy utilizada por los brasileños. Según los datos de la propia plataforma de comunicación social Facebook, alrededor de 127 millones de brasileños acceden la red social, ${ }^{14}$ lo que permite a «Valentina» estar disponible para más de $60 \%$ de la población brasileña, ${ }^{15}$ que utilizando las facilidades de la red social puede conseguir información para solucionar su caso jurídico con el chatbot, y hasta tener el patrocinio de un abogado de la compañía o contratado por ésta.

En la consulta a la OAB/SP número E-4.880/2017, cuyo voto fue comentado en el apartado anterior, el órgano fiscalizador de la profesión afirmó que existen ciertas iniciativas tecnológicas que, con el pretexto de dar soporte a las actividades de defensa, en realidad se prestan para encubrir mecanismos para la mercantilización de la profesión de abogados, o incluso sirven como vehículo de facilitación a la captación indebida de clientela, como el Tribunal Deontológico de São Paulo ya tuvo la oportunidad de comprobar en relación con determinadas consultas. Por lo tanto, las innovaciones tecnológicas dirigidas a la abogacía que confieren carácter mercantilista a la profesión o ayudan e inducen a la captación de clientela, que son minoría, están vedadas, porque ponen en riesgo la seguridad y las protecciones conferidas por el sistema a los destinatarios del derecho, por lo que quedan sin sentido las críticas respecto de que la reglamentación de la profesión sería obstáculo a la evolución de la tecnología en el área.

Por esta razón, y considerando la comprensión que el lector ya tiene sobre el funcionamiento de los chatbots de Hurst Capital explicada anteriormente, no es difícil concluir que esta herramienta se destina a la competencia desleal en forma de capta-

\footnotetext{
14. Filipe Oliveira, «Facebook chega a 127 milhões de usuários mensais no Brasil», Folha de São Paulo, 18 de julio de 2018, disponible en http://bit.ly/2WvulMV.

15. «Projeção da população do Brasil e das Unidades da Federação», Instituto Brasileiro de Geografia e Estatística, disponible en http://bit.ly/2WvVUWz.
} 
ción masiva de clientes. Por ende, hay una herramienta de apoyo profesional, que en verdad es un vehículo de captación disfrazado.

Se suman, entonces, dos problemas para el chatbot «Valentina». El primero es que se trata de una consultoría de oferta al público a través de internet y de forma impersonal, lo cual es vedado por las leyes que regulan la profesión de abogado en Brasil. Segundo, que el chatbot se destina a captar clientes a través de la red social en que se instala, y ofrece una consultoría que terminará —obviamente dentro de los criterios de la empresa - en la captación de potenciales clientes. En una situación como ésta, solamente el equipo de abogado que está por detrás de la empresa gestora de la inteligencia artificial se beneficiará de los clientes captados por la red social, lo que conforma una auténtica competencia desleal y la mercantilización de la profesión de abogado en el país.

Teniendo en cuenta los dos problemas ya analizados y que, según nuestra opinión, vulneran las disposiciones del Estatuto de la OAB y del Código de Ética y Disciplina de la $\mathrm{OAB}$, queda uno más que analizar, y que tiene trascendencia directa en el derecho del trabajo y sus principios rectores, que es la compra de los derechos patrimoniales disponibles (créditos laborales) y las limitaciones de la solución extrajudicial para solucionar los conflictos laborales que representan los principales objetivos de la captación de clientes por los chatbots aquí estudiados.

\section{El posible menoscabo al trabajador en la obtención de los créditos laborales por el uso del servicio de chatbot}

En la introducción de este artículo evidenciamos que la empresa Hurst Capital utiliza la inteligencia artificial - por chatbots - para localizar y adquirir derechos patrimoniales disponibles a través de la cesión de crédito, que es una modalidad de transmisión de la obligación prevista en el Código Civil brasileño. Luego de adquirir los derechos del trabajador, hace negociaciones administrativas y contrata a los abogados para iniciar los procedimientos judiciales contra los deudores en los casos en que sea necesario.

Por ende, la empresa, por medio de su captación (chatbots), una vez identificado un caso con alta probabilidad de retorno financiero y buena rentabilidad en el suceso del certamen, determinará si es un caso de judicialización - situación en que contratará los abogados para el trabajo- o utilizará una solución extrajudicial de los conflictos.

La empresa entró en el mercado ofreciendo un servicio que cubre los costos de litigación en un momento en que Brasil vive la incertidumbre de los efectos de la reforma laboral del año 2017 (Ley 13.467/2017), cuyas disposiciones procesales sobre las costas judiciales resultaron en la disminución de las demandas laborales en aquel país, como comentamos en la introducción de este trabajo. 
En este punto, es fundamental comprender que en Brasil hay un intervencionismo estatal en la determinación de los derechos mínimos de los trabajadores. Más allá, hay un derecho constitucional del trabajo que consta en los artículos 7, 8, 9, 10 y 11 de la Constitución de la República Federativa de Brasil de 1988, que por fuerza del artículo 60, sección 4 de la misma Carta Política, siquiera puede ser objeto de deliberación para reforma vía enmienda constitucional, ya que estos derechos sociales del trabajador son verdaderas cláusulas pétreas constitucionales.

La existencia de un orden público laboral consagra un principio conocido por los laboralistas de América Latina — gracias a las enseñanzas del profesor Américo Plá Rodríguez-, el principio de la irrenunciabilidad de los derechos laborales (Plá Rodríguez, 2015: 125). Consecuentemente, la doctrina brasileña aboga por la indisponibilidad de los derechos del trabajo, por el hecho de que existe una verdadera limitación a la autonomía de la voluntad por la tutela de la parte hiposuficiente concedida por el ordenamiento jurídico (Monteiro de Barros, 2009: 186).

Este principio, además, está positivado en la Consolidación de las Leyes del Trabajo (CLT, Decreto Ley 5.452 de 1943) en sus artículos $9,{ }^{16} 444^{17}$ y $448 .{ }^{18}$ Por estos dispositivos, la doctrina dominante en el país no admite la renuncia de derechos antes ni durante el contrato de trabajo, mientras que es más aceptada la posibilidad de renuncia después de la extinción del contrato laboral (Bezerra Leite, 2018: 107).

Un punto interesante con respecto de la terminación del contrato de trabajo es que antes la renuncia al empleo y el recibo de pago del finiquito para trabajadores con más de un año de servicio solo eran válidos si comprobasen la asistencia del sindicato o si fuera hecho frente a una autoridad del Ministerio del Trabajo y de la Previsión Social (artículo 477, sección 1 de la CLT brasileña antes de la Reforma Laboral de la Ley 13.467 de 2017). Sin embargo, ahora, con la reforma del año 2017, el párrafo primero del artículo 477 fue derogado, lo que nos lleva a entender que el recibo del finiquito para cualquier trabajador tiene validez, aunque sin la intervención del sindicato o de una autoridad del Ministerio del Trabajo y de la Previsión Social.

Obviamente, el acto de firma del finiquito sigue siendo anulable en la justicia especializada laboral, sea por incapacidad del agente, error, fuerza y dolo, simulación o fraude a la ley (Carrion, 2018: 478). Empero, la quitación de la asistencia en el acto de

16. «Serán nulos de pleno derecho los actos practicados con el objetivo de desvirtuar, impedir o fraudar la aplicación de los preceptos contenidos en la presente Consolidación».

17. «Las relaciones contractuales de trabajo pueden ser objeto de libre estipulación de las partes interesadas en todo lo que no contravenga a las disposiciones de protección al trabajo, a los contratos colectivos que les sean aplicables y a las decisiones de las autoridades competentes».

18. «En los contratos individuales de trabajo solo es lícita la modificación de las respectivas condiciones por mutuo consentimiento, y aun así siempre que no resulten, directa o indirectamente, en perjuicios al empleado, so pena de nulidad de la cláusula vulneradora de esta garantía». 
renuncia al empleo permitirá situaciones de renuncia forzada y, aunque el término del contrato de trabajo será menos engorroso por la ausencia de necesidad de intervención en el finiquito de trabajadores con más de un año, eso igualmente podría prestarse para irregularidades en el pago. Una situación que no es deseada por los trabajadores que tendrán que recurrir a la justicia laboral, con la incertidumbre de si podrán ser condenados en costas judiciales. Esta situación favorece mucho más el nicho de mercado explotado por la empresa Hurst Capital.

Si hablamos de lo que puede hacer el trabajador que en el término de su relación de trabajo tiene derechos patrimoniales a su disposición o si estos derechos le son negados, habrá que distinguir en este momento la renuncia de la transacción y la transacción de la conciliación, para poder comprender los límites en la actuación de la Hurst Capital con el uso del chatbot.

La renuncia se refiere más bien a un derecho ya reconocido, incuestionable, en que el renunciante dispone de su derecho a cambio de nada (Bezerra Leite, 2018: 107). La renuncia es una abdicación que el titular hace de su derecho, sin transferirlo a nadie más (Silva Pereira, 2009). Por esta razón, en el derecho del trabajo la renuncia tiene un campo de aplicación reducido, por el hecho de que el legislador abandonó el principio de la igualdad de derechos o igualdad formal para acercarse a la igualdad de hecho o igualdad material, lo que generó un desequilibrio jurídico para compensar una desigualdad entre las partes que componen una relación de trabajo (Monteiro de Barros, 2009: 197-198). La renuncia debe ser siempre expresa (Monteiro de Barros, 2009: 199) y será permitida en la ruptura del contrato, es decir, con la extinción de la relación de trabajo (Monteiro de Barros, 2009: 202).

La transacción, al revés, presume la controversia, la duda, la incertidumbre del derecho. En este caso, cada parte dispone de algo que le pertenece (Bezerra Leite, 2018: 107). En la transacción ambas partes hacen concesiones recíprocas, naciendo de ahí el derecho de acción para los transigentes (Monteiro de Barros, 2009: 205). La transacción extrajudicial es completamente posible; sin embargo, tampoco tendrá la fuerza de cosa juzgada, pudiendo ser objeto de una demanda judicial futura, por lo cual es entonces anulable por incapacidad del agente, error, fuerza y dolo, simulación o fraude a la ley.

$\mathrm{Al}$ «comprar la pelea» del trabajador por medio del chatbot, la empresa responsable podría intentar utilizar el mecanismo extrajudicial de solución de controversias, y nada garantiza que la transacción obtendrá el mejor resultado para los trabajadores, que ya se sienten lo suficientemente inhibidos para poder iniciar una demanda en la justicia especializada laboral. Esta situación puede prestarse a abusos por parte de la empresa mediadora en favor del empleador y difícilmente obtendremos estadísticas precisas de la solución de sus casos, sobre todo extrajudiciales, para poder verificar la eficiencia en la recuperación de créditos laborales para el trabajador y comparar con demandas similares en los tribunales laborales para hacer estas afirmaciones con mayor certeza. 
Además, los propios trabajadores no siempre conocen sus derechos lo suficientemente bien como para saber que una transacción extrajudicial le da el derecho a judicializarla siempre y cuando descubra que fue vulnerado en este procedimiento.

La transacción de créditos laborales, que implican derechos previstos en la ley, solo tiene fuerza de cosa juzgada si es hecha en la Justicia del Trabajo (artículo 831, párrafo único de la CLT brasileña), ${ }^{19}$ cuando se lleva a cabo en un acuerdo formalizado ante el juez del trabajo; recién entonces vale como decisión irrecurrible. Esta transacción hecha en la justicia especializada laboral se llama conciliación y es obligatoria al inicio de la audiencia so pena de nulidad del obrado en juicio (Carrion, 2018: 722).

Dado todo lo argumentado, se vislumbra la existencia de algunas situaciones en que puede haber una lesión o vulneración de los derechos del trabajador en la gestión de recuperación de sus créditos laborales, generados por el actual escenario de incertidumbre en Brasil y su reforma laboral del año 2017.

La empresa Hurst Capital entra en el mercado con una herramienta que promete la recuperación de los derechos patrimoniales del trabajador, captando clientes masivamente por una consultoría informal en las redes sociales. Esta situación de doble vulnerabilidad - considerando el trabajador como parte hiposuficiente del contrato de trabajo y considerando el miedo que la reforma laboral brasileña ha causado en los posibles litigantes frente al nuevo sistema de costas judiciales- es preocupante, y puede prestarse para que los trabajadores vean sus derechos menoscabados.

\section{Conclusiones}

La propuesta de este artículo fue estudiar el caso de la consultoría laboral por un chatbot de inteligencia artificial nombrado «Valentina, el robot del trabajador» y discutir sus posibles consecuencias éticas y profesionales en Brasil, además de sus implicaciones en materia laboral.

Los puntos a ser cuestionados fueron: i) la consultoría laboral por una inteligencia artificial, que según el Estatuto de la Abogacía de Brasil alude a la exclusividad de esta actividad por un profesional habilitado al ejercicio de la profesión; ii) la captación de acciones por medio de las redes sociales; y iii) el posible menoscabo al trabajador en la obtención de los créditos laborales que les son debidos por la transferencia de parte o la totalidad de la indemnización a la empresa contratada, o por el uso de la solución extrajudicial del conflicto.

En cuanto al primer punto, aunque no se puede afirmar que la empresa está incurriendo en una contravención penal por el ejercicio ilegal de la profesión de abogado, y por estar de acuerdo con que el uso de la inteligencia artificial en la abogacía abre

19. «En caso de conciliación, el término que se emite valdrá como decisión irrecurrible, salvo para la previsión social en cuanto a las cotizaciones que le son debidas». 
un nuevo nicho de mercado para los profesionales jurídicos dedicados a la lógica jurídica -como forma de implementación de las argumentaciones válidas en materia de inteligencia artificial, haciéndola funcionar con inferencias dentro de la lógica de funcionamiento de un sistema jurídico en especial- concluimos sobre este primer punto que la consultoría jurídica no puede considerarse como una oferta impersonal al público, menos si utiliza medios de comunicación como internet, y en este caso, las redes sociales. Por esta razón, el chatbot «Valentina» vulnera las garantías del Estatuto de la OAB y del Código de Ética y Disciplina de la OAB en este primer punto.

En segundo lugar, no hay dudas de que existe una clara intención de captar clientes con el uso de un chatbot en una red social que alcanza a más del 60\% de la población brasileña. De esta forma, esta herramienta no se destina a facilitar el trabajo de un grupo de abogados, sino a identificar demandas rentables y poco temerarias para que la empresa lucre con el resultado de su gestión judicial o extrajudicial.

$\mathrm{El}$ «robot del trabajador» termina siendo un instrumento de marketing y captación que favorece solamente al grupo de abogados subcontratados por la empresa dueña de la inteligencia artificial. De esta forma, no se trata de una plataforma de apoyo al profesional abogado, no es una innovación que facilita su propio trabajo, sino que es un instrumento de captación de clientes rentables. Nuevamente, el chatbot «Valentina» vulnera las garantías del Estatuto de la OAB y del Código de Ética y Disciplina de la $\mathrm{OAB}$ en este segundo punto.

Por fin, y por ser un punto sensible en cuanto al uso de una herramienta de captación de demandas destinada a trabajadores que sufren el menoscabo o la lesión de sus derechos laborales, existe una alta probabilidad de exponer a los trabajadores - que son la parte hiposuficiente del contrato de trabajo- a una nueva situación de inseguridad.

La reducción de demandas en los tribunales especializados laborales de Brasil, por cuenta de la reforma laboral promovida por la Ley 13.467 del año 2017, terminó por favorecer al nicho explotado por Hurst Capital. Esto porque, al ofrecer un servicio que cubre los costos de la demanda, ella podrá captar muchos más clientes que no tendrán el temor de pagar las costas judiciales en situaciones que antaño no le corresponderían.

No obstante, no sabemos cuánto la empresa cobra por estos servicios, teniendo en cuenta que en la práctica los profesionales jurídicos cobran entre $20 \%$ a $30 \%$ del resultado del litigio (en promedio). Además, existe la posibilidad de una solución extrajudicial del conflicto, que puede llevar a una transacción no tan favorable para el trabajador que, por veces, ni siquiera sabe que puede cuestionar el resultado de la transacción en la justicia laboral, ya que la conciliación frente a un magistrado del trabajo es la única forma de hacer irrecurrible lo acordado por las partes (empleador y trabajador). 
Frente a todo lo expuesto, es poco aconsejable la explotación de este mercado por Hurst Capital, teniendo en cuenta que los trabajadores son los que tienen menos poder de negociación y que sufren la mayor vulneración de sus derechos en una relación de trabajo. Aprovecharse del miedo a la judicialización de los conflictos laborales por una reforma flexibilizadora y que, en algunos puntos, representa un verdadero retroceso social, es poco ético y no representa el eje de la abogacía, que es la administración de la justicia y la persecución de los valores éticos y morales en su actuación.

La tecnología es imprescindible para los avanzos sociales, y viene facilitando el ejercicio de las profesiones y la explotación de la industria de una forma antes inimaginable. Sin embargo, no puede prestarse como un instrumento de explotación sin medidas y que ponga en peligro la seguridad jurídica y la ética profesional.

A partir del momento en que paso a pensar en un cliente como una posibilidad de lucro, y utilizo la tecnología para prever mis potenciales pérdidas y ganancias, la abogacía deja de ser la persecución de la justicia para volverse un negocio rentable. Ya es poco ético elegir el cliente por el valor del retorno financiero que me pueda proporcionar con su conflicto - para no decir problema-. Pero, es aún menos ético cazar demandas rentables con una herramienta estricta que puede generar mucho más daño en su camino.

\section{Reconocimiento}

Este artículo fue desarrollado en el marco del proyecto DIUFRO núm. DIo108.

\section{Referencias}

Arese, César, Luiz Eduardo Gunther y Manoel Carlos Toledo Filho (2017). «Las reformas laborales de Brasil y Argentina: Un sordo ruido». Revista eletrônica [do] Tribunal Regional do Trabalho da $9^{a}$ Região, 7 (63): 219-225. Disponible en https:// hdl.handle.net/20.500.12178/122549.

BAroni, Robison (2009). Ética na advocacia. São Paulo: LTr.

Benítez, Raúl, Gerard Escudero, Samir Kanaan y David Masip Rodó (2014). Inteligencia artificial avanzada. Barcelona: UOC.

Bezerra Leite, Carlos Henrique (2018). Curso de direito do trabalho. 10. ${ }^{a}$ ed. São Paulo: Saraiva.

Carrion, Valentin (2018). CLT: Comentários à consolidação das Leis Trabalhistas. Actualizado por Eduardo Carrion. São Paulo: Saraiva.

Konar, Amit (1999). Artificial intelligence and soft computing: Behavioral and cognitive modeling of the human brain. Nueva York: CRC Press.

LôBo, Paulo (2007). Comentários ao Estatuto da Advocacia e da OAB. 4. ${ }^{\text {a }}$ ed. São Paulo: Saraiva. 
Martinez, Luciano (2018). Reforma trabalhista: Entenda o que mudou. 2. ${ }^{a}$ ed. São Paulo: Saraiva.

Monteiro de Barros, Alice (2009). Curso de direito do trabalho. 5. ${ }^{a}$ ed. São Paulo: LTr.

Moraes, Alexandre (2003). Direito constitucional. 13. ${ }^{a}$ ed. São Paulo: Atlas.

Piovezan, Giovani Cássio y Gustavo Oliveira Freitas (2015). Estatuto da Advocacia e da OAB comentado. Curitiba: OAB Paraná.

Plá Rodríguez, Américo (2015). Los principios del derecho del trabajo. Montevideo: Fundación de Cultura Universitaria.

Silva Pereira, Caio Mário da (2009). Instituições de direito civil: Introdução ao direito civil. Teoria geral de direito civil. Volumen 1. Río de Janeiro: Forense.

Souza Júnior, Antonio Umberto de, Fabiano Coelho de Souza, Ney Maranhão y Platón Teixeira de Azevedo Neto (2018). Reforma trabalhista: Análise comparativa e crítica da Lei no 13.467/2017 e da Med. Prov. $n^{\circ}$ 808/2017. São Paulo: Rideel.

Walton, Douglas (2005). Argumentatio methods for artificial intelligence in law. Heidelberg: Springer.

\section{Sobre el autor}

Rodrigo Monteiro Pessoa es abogado y administrador. Graduado en derecho por el Instituto de Educación Superior de Paraíba, Brasil, y en administración por la Universidad Federal da Paraíba, Brasil. Magíster en derecho económico por la Universidad Federal de Paraíba. Doctor en derecho por la Universidad de Chile. Académico de la Universidad de la Frontera, Chile. Su correo electrónico es rodrigo.pessoa@ ufrontera.cl. (1D) http://orcid.org/oooo-0o02-3393-4139. 


\title{
REVISTA CHILENA DE DERECHO Y TECNOLOGÍA
}

La Revista de Chilena de Derecho y Tecnología es una publicación académica semestral del Centro de Estudios en Derecho Informático de la Facultad de Derecho de la Universidad de Chile, que tiene por objeto difundir en la comunidad jurídica los elementos necesarios para analizar y comprender los alcances y efectos que el desarrollo tecnológico y cultural han producido en la sociedad, especialmente su impacto en la ciencia jurídica.

\author{
EDITOR GENERAL \\ Daniel Álvarez Valenzuela \\ (dalvarez@derecho.uchile.cl) \\ SITIO WEB \\ rchdt.uchile.cl \\ CORREO ELECTRÓNICO \\ rchdt@derecho.uchile.cl \\ LICENCIA DE ESTE ARTÍ́CULO \\ Creative Commons Atribución Compartir Igual 4.o Internacional
}

\begin{abstract}
La edición de textos, el diseño editorial
y la conversión a formatos electrónicos de este artículo

estuvieron a cargo de Tipográfica

(www.tipografica.cl).
\end{abstract}

